\title{
A broader palette for luciferase
}

Engineered firefly enzymes use new substrates and emit at redder wavelengths.

Reporters such as green fluorescent protein and luciferase literally illuminate cells' innermost activities, translating gene expression into light. Compared to fluorescent reporters, luciferase has the advantage that no external light source is required to reveal its presence. However, this enzyme has its own disadvantages. Luminescence depends on delivery of the enzyme's substrate-luciferin-into cells; a relatively high concentration of luciferin is required to produce sustainable levels of light; and the reaction produces bluish wavelengths that are not ideal for in vivo imaging.

Researchers led by Stephen Miller at University of Massachusetts Medical School had previously synthesized analogs of luciferin in the hopes of improving the luminescence imaging qualities. These aminoluciferin analogs produce a bright burst of light in the presence of luciferase, but their emission levels quickly subside. Reasoning that the reaction products must inhibit the enzyme, Miller's team set about to remodel the luciferase binding pocket to allow the products to dissociate more easily.

They made several luciferase mutants, tested their emission when exposed to a variety of synthesized aminoluciferins in vitro, and then tested them again in mammalian cells and cell lysates. This yielded a mutant enzyme that performs better with aminoluciferins than the wild-type enzyme does. The light produced was sustained over longer periods and was redder, both desirable properties for many imaging applications.

The tests also revealed some optimal pairings of engineered luciferases and aminoluciferins. Intriguingly, several of the engineered luciferases preferred one substrate over another, raising the possibility that separate enzymes could be used in the same cell to illuminate different processes. For example, separate luciferases could be used to monitor expression of different genes. And new versions of luciferins could have other desirable properties, such as producing red-shifted wavelengths or permeating cells more easily, says Miller, who is working on making enzymes even more selective for different substrates.

This kind of work shows that the scope of bioluminescent imaging is more expansive than many researchers assume. "The palette can be broadened," says Miller. In time, he says, luminescent imaging resources could be "more like what you see with fluorescence; you can have multiple wavelengths and multiple probes."

\section{Monya Baker}

\section{RESEARCH PAPERS}

Harwood, K.R. et al. Identification of mutant firefly luciferases that efficiently utilize aminoluciferins. Chem. Biol. 18, 1649-1657 (2011). 